https://doi.org/10.7203/Normas.3.4673

\title{
EL LÉXICO DEL TURISMO EN EL ESPAÑOL DE ARGENTINA: ESTUDIO DE LAS PRINCIPALES PÁGINAS WEB HOTELERAS*
}

\author{
THE LEXICON OF THE TOURISM IN THE SPANISH OF ARGENTINA: STUDY OF \\ THE MAIN HOTEL WEBSITES
}

\author{
David GIMÉNEZ FOLQUÉS \\ Universitat de València
}

\section{RESUMEN:}

El mundo del turismo ha evolucionado en los últimos tiempos de una manera notable gracias al auge de internet. Asimismo, con esta nueva herramienta, este sector ha ampliado su campo de visión comercial y, por lo tanto, ha encontrado una vía más rápida y eficaz para llegar al público.

El ámbito de investigación lingüística que se dedica al turismo no ha tardado en hacerse eco de esta nueva situación y, de esta manera, ha empezado a contemplar en su campo de estudio la variante que supone internet en el turismo. Uno de los motivos de este interés es el aumento terminológico que se ha detectado en el ámbito turístico mediante esta vía. Este incremento se debe a la aparición masiva de anuncios turísticos por la facilidad y rapidez que supone, por parte de las empresas y demás entidades, situarlos en la web.

En este artículo, estudiaremos en concreto el español turístico de Argentina, centrándonos en el aspecto léxico. Tomaremos especial atención en aquel léxico que es propio del habla argentina, para observar así sus características y peculiaridades. Para llevar a cabo el estudio de estos parámetros léxicos utilizaremos el filtro de los diccionarios académico, tanto de los españoles como de los argentinos.

PALABRAS CLAVE: turismo, lengua española, web hotelera, léxico, extranjerismos.

\section{ABSTRACT:}

The world of tourism has evolved in recent times because of the impact of the Internet. The tourism enterprises have expanded their commercial point of view and have found a faster way in reaching clients through increased tourism advertising on the Web. Along with this increase in advertising are the opportunities to study the lexicon of tourism. In this article, we will study the Spanish of Argentina focusing on the lexical aspect.

KEY WORDS: Tourism, Spanish language, hotel website, lexicon, foreign words.

* Este trabajo se adscribe al proyecto: Análisis léxico y discursivo de corpus paralelos y comparables (español-inglés-francés) de páginas electrónicas de promoción turística, referencia FFI2011-24712 (Ministerio de Economía y Competividad), dirigido por Julia Sanmartín.

NORMAS. REVISTA DE ESTUDIOS LINGÜÍSTICOS HISPÁNICOS, NÚMERO 3 (AÑO 2013):

http://www.uv.es/normas

(ISSN 2174-7245) 


\section{ESTADO DE LA CUESTIÓN}

El léxico del turismo está caracterizado por la perspectiva internacional que tiene el sector turístico. La difusión del turismo es indiscutible, y su manera de expresarse ha ido evolucionando hasta derivar en diferentes géneros discursivos. Según señala Calvi (2010: 9), estos géneros discursivos se dividen en tres grupos: géneros propios de la gestión, géneros propios de la descripción y promoción de lugares turísticos, y, finalmente, aquellos derivados de su difusión en internet:

Se tomarán en consideración tanto los géneros propios de la gestión (contratos de viaje, normativas, etc.) como los creados para la descripción y promoción de los lugares turísticos (guía, folleto, etc.). Asimismo, se tendrá en cuenta la aparición de nuevos géneros difundidos a través de Internet, en los que los usuarios se convierten en expertos de la comunicación turística (foros, blogs, etc.), creando nuevos paradigmas comunicativos que podrían trastocar los esquemas tradicionales.

Además, la incursión del turismo como actividad profesional ha aumentado la difusión de algunos de estos géneros, como sería el caso de la guía, el folleto o el programa de viaje. Hay que tener en cuenta que en el ámbito turístico se inserta léxico de otros sectores como, por ejemplo, el gastronómico, el geográfico o el histórico. Este fenómeno ha influido a la hora de categorizar los géneros derivados del turismo y ha dificultado la clasificación terminológica del léxico turístico.

Según Calvi (2010) esta mezcla terminológica puede haber sido un factor determinante a la hora de que el léxico del turismo haya tenido menos transcendencia científica que otros lenguajes de especialidad. Sin embargo, Cronin (2000) mantiene que el resultado terminológico que deriva del intercambio léxico en este sector ha sido la clave en la formación del fenómeno turístico y en el intercambio cultural que ha provocado. Esto se debe a que este tipo de textos van dirigidos a público de diferentes nacionalidades y pretenden atraerlo, además de con unas instalaciones adecuadas y unos precios atractivos, dando a conocer la cultura del lugar y haciéndola llamativa para el usuario.

Por otro lado, el factor de traducción también ha sido beneficioso desde el punto de vista del estudio científico del texto turístico como lenguaje de especialidad. La inclusión del turismo en el ámbito profesional, junto con la necesidad de promocionarse a lo largo de las diferentes nacionalidades, ha creado la necesidad de traducir los textos a aquellos idiomas que eran el objeto de sus textos ${ }^{1}$.

Estos aspectos positivos, pese a su diversidad léxica, han provocado que en los últimos años haya habido más aproximaciones linguiísticas hacia este lenguaje de especialidad, no solo en la variante española peninsular, sino también en la americana, además, por supuesto, de otros idiomas de prestigio como el inglés.

\footnotetext{
${ }^{1}$ Estos idiomas variarán según el interés: prestigio, cercanía geográfica, afinidad, etc.
} 
Entre los estudios actuales sobre el turismo en español ${ }^{2}$, podemos destacar los de Alcaraz (2006); Aragón (2009), Calvi (2006a, 2010 y 2011) Gallego y Peyrolón (2009) o Sanmartín (2011a y 2012). Muchas de estas obras basan sus trabajos en la traducción o en la comparación del español con otras lenguas. Por ejemplo, Alcaraz (2009) propone una minuciosa clasificación del léxico con mayor uso en el ámbito turístico en el área de gestión y descripción de lugares. Engloba este léxico en un diccionario de términos de turismo y de ocio entre el español y el inglés. Se centra en cuatro sectores del turismo: el ocio, el viaje, la restauración y el alojamiento. Aragón (2009), por otro lado, también realiza un diccionario de términos del turismo pero, en este caso, entre el español y el francés. Resalta en su aportación el especial cuidado con el que trata los préstamos en ambas lenguas en el ámbito turístico, ya que incluye las posibles adaptaciones ortográficas y fonológicas de estos préstamos y las recomendaciones que hacen para su traducción o adaptación los organismos académicos.

Hay que resaltar la labor de Maria Vittoria Calvi que ha centro los últimos años de su investigación en analizar el lenguaje turístico como lenguaje de especialidad. Además, ha sabido analizar y clasificar los distintos géneros discursivos que derivan de este ámbito. La autora ha señalado la importancia que está tomando la profesionalización del texto turístico, como hemos señalado anteriormente, y su inserción en internet.

Destacables también son los trabajos de Julia Sanmartín Sáez, que contempla el factor de variación dentro de la lengua española, concretamente entre la variedad peninsular y la americana, España y Chile. La autora destaca que pese a la variedad lingüística se puede observar una unidad lingüística (Sanmartín, 2011a: 355):

En general, se podría concluir que en este tipo de discurso del español del turismo hay más unidad que diversidad. Sin embargo, más allá de la necesaria (y aquí en especial) tendencia uniformizadora que se exige a un producto que debe cumplir unas normativa (como las medidas de una cama o las clasificaciones hoteleras), se percibe un intento de atraer al posible cliente con un léxico considerado como más novedoso e innovador; y por ello, se prefiere pernoctar en una single que en una habitación individual.

De hecho, esta es la hipótesis de la que vamos a partir en este trabajo, ya que observaremos si se cumplen los mismos patrones entre el léxico turístico de España y el de Argentina. Además, Sanmartín (2012) realiza una aproximación analítica hacia los blogs, los foros, los libros de visita o las páginas web privadas e institucionales de promoción de hoteles. Todo ello es vinculado al fenómeno que supone internet, elemento que hay que tener en cuenta, ya que cada vez más todos estos estudios, además de estudiar las guías turísticas y los folletos, han empezado a interesarse por las páginas web, dada la difusión que está teniendo el texto turístico en internet y la facilidad que supone acercarse a estos textos y analizarlos en la web.

\footnotetext{
${ }^{2}$ Obras propiamente en español o en comparación con otras lenguas.
} 
Por ello, en el trabajo que presentamos a continuación, además de recoger la buena labor de estos trabajos y la de aquellos que no hemos mencionado, pero que también han sido clave en el desarrollo del texto turístico, vamos a centrarnos en dos factores, variación lingüística (entre el español de Argentina y el de España) y difusión del texto turístico en internet (mediante la extracción de un corpus en internet procedente de algunas páginas web de hoteles).

\section{OBJETIVOS Y METODOLOGÍA}

En el presente artículo nos proponemos analizar las particularidades del español de Argentina en el léxico turístico con respecto al español peninsular. Para ello, tomaremos como referencia las páginas web hoteleras dentro de una pertinente selección de hoteles de todas las categorías. A partir de esta selección léxica realizaremos una base de datos que analizaremos, a continuación, según el léxico extraído.

El análisis del léxico será el segundo paso de este trabajo. Para realizar una clasificación significativa de los vocablos extraídos, usaremos el filtro de los diccionarios académicos seleccionados. En este caso el Diccionario de la lengua española (vigésima segunda edición) y el Diccionario panhispánico de dudas, por un lado, y el Diccionario del habla de los argentinos y El gran diccionario de los argentinos $^{3}$ por el otro. El objetivo de este análisis es observar las peculiaridades del léxico turístico argentino y señalar cómo lo recogen los diccionarios académicos del español en general y del español argentino en particular.

Para este análisis habrá que tener en cuenta que la lengua española presenta una variación geográfica y que esta variación, en este caso propia de la zona argentina, afectará al discurso turístico, y en concreto, al léxico. Muchas de las conclusiones ya predeterminadas de este artículo partirán del proyecto: Análisis léxico y discursivo de corpus paralelos y comparables (español-inglés-francés) de páginas electrónicas de promoción turística, referencia FFI2011-24712, proyecto en el que se inserta este trabajo.

En definitiva, se va a realizar un estudio léxico, prestando especial importancia a los términos ${ }^{4}$ turísticos propios de Argentina y recogidos solamente por diccionarios argentinos, a las voces recogidas por diccionarios del español general con la marca de «americanismo», y, finalmente, a los extranjerismos 5 utilizados.

\footnotetext{
${ }^{3}$ Versión digitalizada por el periódico Clarín.

${ }^{4}$ Pese a emplearse la voz «término» no nos referimos a léxico de especialidad, sino que se alude, generalmente, a voces genéricas del léxico turístico.

${ }^{5}$ Se entiende como extranjerismo una voz que procede de otra lengua y que es utilizado en la lengua de destino, en su forma original o adaptada a su sistema lingüístico.
} 
1. 1. El corpus argentino seleccionado: páginas web de comercialización hotelera privada

En primer lugar, se han tomado como corpus argentino de referencia cincuenta páginas web que contienen promoción de hoteles de Argentina. En la clasificación siguiente se ha utilizado una serie de criterios para su adscripción al corpus. El criterio de ordenación ha sido temporal, es decir, por orden de búsqueda. En primer lugar, aparece el nombre completo del hotel; a continuación, aparece la ciudad a la que pertenece y, finalmente, la categoría del hotel. Alto significa que el hotel tiene cuatro o cinco estrellas, medio contempla las tres y las dos estrellas y, en último lugar, bajo se refiere a hoteles de una estrella:

1. Alma suites (Bariloche), alto;

2. Aspen Square Hotel (Buenos Aires), alto;

3. Ayuntun Hue Apart Hotel (Buenos Aires), medio;

4. Diplomatic Hotel Mendoza (Mendoza), alto;

5. Gran Hotel Hispano (Buenos Aires), medio;

6. Hotel Benevento (Buenos Aires), medio;

7. Hotel Tres Reyes (Bariloche), medio;

8. Hotel Mendoza (Mendoza), medio;

9. Hotel Venus (Mendoza), medio;

10. Hotel Sienna (Mendoza), bajo;

11. Hotel Casa Real (Salta), alto;

12. Hotel Satiri (Salta), bajo;

13. Hotel Regidor (Salta), medio;

14. Hotel Guermes (Salta), medio;

15. Hotel Design Suites (Salta), alto;

16. Hotel Xelena (El Calafate), alto;

17. Hostería Sir Thomas (Salta), bajo;

18. Hotel Mustapic (Ushuaia), bajo;

19. Hotel de los Andes (Ushuaia), medio;

20. Hotel Antartida Argentina (Ushuaia), medio;

21. Hotel Campanilla (Ushuaia), medio;

22. Hotel Cap Polonio (Ushuaia), medio;

23. Hotel Rosa de los Vientos (Ushuaia), medio;

24. Hotel Austral Kauyeken (Ushuaia), medio;

25. Hotel Lennox Ushuaia (Ushuaia), alto;

26. Hotel Cauquenas (Ushuaia), alto;

27. Hotel Gran Vía (Buenos Aires), bajo;

28. Hotel Prince (Buenos Aires), bajo;

29. Hotel Mundial (Buenos Aires), medio;

30. Hotel Etoile (Buenos Aires), alto; 
31. Hotel Reconquista Luxor (Buenos Aires), alto;

32. Hotel Boca Juniors Design (Buenos Aires), alto;

33. Hotel Tierra Colorada (Puerto Iguazú), bajo;

34. Hotel Boutique de la Fonte (Puerto Iguazú), alto.

35. King's Hotel Bariloche (Bariloche), medio;

36. Koten Hotel (Buenos Aires), medio;

37. La Casa del Peregrino (Salta), bajo;

38. La Posada del fin del mundo (Ushuaia), bajo;

39. Las Hayas Resort Hotel (Ushuaia), alto;

40. Mio Buenos Aires (Buenos Aires), alto;

41. Nuñez Suites (Buenos Aires), bajo;

42. Petit Hotel Mi Capitan (Puerto Iguazú), medio;

43. Pop Hostel Garden (Puerto Iguazú), medio;

44. Posada Angelus (Salta), bajo;

45. Room Mate Carlos (Buenos Aires), alto;

46. Serena Hotel Buenos Aires (Buenos Aires), alto;

47. South Beach (Buenos Aires), alto;

48. Tango Lodge (Buenos Aires), medio;

49. Two Hotel (Buenos Aires), medio;

50. Yreta Apart Hotel (Puerto Iguazú), medio.

Se ha contemplado la variedad tanto en la ciudad de pertenencia del hotel como en la categoría del mismo. Esta variedad es importante para dar cabida a las diferentes pautas geográficas y categoriales que los hoteles podían ofrecer. Además de estas pautas, como vamos a ver a continuación, hay otros factores que influyen a la hora de seleccionar un término u otro.

Evidentemente, en el léxico utilizado en una web hotelera, como señala Sanmartín (2011a: 337), predomina un objetivo que incide directamente en el léxico utilizado:

No obstante, comparten el mismo objetivo: persuadir al lector para que viaje al lugar y adquiera una de sus promociones. Y, sin duda, uno de los productos que intentan ofertar es el posible establecimiento hotelero. Se concibe, pues, como un discurso dirigido a un público no especialista en gestión hotelera y con un predominio de la función persuasiva.

Esta función persuasiva provoca que el léxico utilizado pretenda captar el mayor número de clientes posibles y a que estos accedan a contratar alguno de sus servicios. Por ello, muchas veces se seleccionan términos que llamen la atención, como, por ejemplo, los extranjerismos, especialmente los anglicismos ${ }^{6}$.

${ }^{6}$ En algunas ocasiones, el uso de un extranjerismo puede derivar en un toque de distinción respecto a la palabra patrimonial conocida. También puede formar parte de una estructura uniformada.

NORMAS. REVISTA DE ESTUDIOS LINGÜÍSTICOS HISPÁNICOS, NÚMERO 3 (AÑO 2013):

http://www.uv.es/normas

(ISSN 2174-7245) 
Hemos seleccionado las webs privadas hoteleras por la difusión de información que conllevan y por la variedad, como hemos señalado al principio, que podemos encontrar dentro de los hoteles tanto en el factor geográfico como en el categorial.

\section{2. Filtros de contraste}

En principio, el discurso de las páginas web hoteleras de Argentina no se dirige exclusivamente a un público argentino, sino que por el tipo de información ofrecida se trata o bien de un receptor de otros países de Hispanoamérica, o bien de España, ya que el objetivo es llegar al máximo número de clientes posible. Por ello, se utiliza un discurso estándar dentro del ámbito hispánico con las particularidades obvias del español de Argentina.

Hay que tener en cuenta que este estudio se realiza desde la perspectiva del español peninsular, por ello, se extraerán las particularidades propias del español argentino en el discurso turístico. Así pues, revisaremos estas páginas web hoteleras y extraeremos todas aquellas voces que presenten algún elemento de uso diferencial respecto del español peninsular.

Como hemos señalado en la introducción, para catalogar estas voces diferenciales utilizaremos varios filtros divididos en dos grupos:

1. diccionarios propios del español general;

2. diccionarios propios del español argentino ${ }^{7}$.

En el primero grupo, utilizaremos el Diccionario de la lengua española (vigésima segunda edición) y el Diccionario panhispánico de dudas ${ }^{8}$. Evidentemente, en este diccionario se contempla tanto el español peninsular como el americano. Cuando un término hace referencia a un elemento propio del español americano, el Diccionario marca esta voz con la abreviación $A m{ }^{9}$. Más aún, cuando es propio de Argentina, lo marca con la abreviación Arg. ${ }^{10}$. Aunque es un diccionario que engloba todo el ámbito hispánico, parte de la perspectiva peninsular, ya que en el caso de seleccionar un término propio del español peninsular, este no aparece con ningún tipo de marca. De esta manera damos un primer paso en la diferenciación léxica del turismo entre el español peninsular y el argentino.

Un segundo paso aparece enmarcado en el segundo filtro; es decir, hay algunas palabras que no aparecen en ninguno de los dos diccionarios del español panhispánico señalados anteriormente. En este caso se tuvo que recurrir a diccionarios propios del

\footnotetext{
7 También se ha consultado el Diccionario de americanismos de la Real Academia Española (2010) cuando ha sido conveniente; ya que en los corpus de palabras buscábamos o bien diccionarios que partieran de la perspectiva peninsular, o bien diccionarios que fueran específicos del español argentino.

${ }^{8}$ Especialmente, para los casos de extranjerismos; ya que supone una de las obras que con más rigor los ha recogido.

${ }^{9}$ Americanismo.

${ }^{10}$ Argentina.
} 
español argentino para observar si aquí sí tenían cabida. Para este propósito seleccionamos el Diccionario del habla de los argentinos y El gran diccionario de los argentinos. En primer lugar, comparamos las palabras que aparecían en los diccionarios generales, con el propósito de observar cómo aparecían definidas en estos diccionarios, sobre todo por si aparecían con una nueva acepción. En segundo lugar, dábamos paso a la búsqueda de aquellos términos que no aparecían en los diccionarios generales. Evidentemente, una palabra que solamente aparecía en un diccionario propio del español argentino ya nos mostraba su diferenciación con respecto al español peninsular.

De esta manera, se decidió agrupar los resultados obtenidos del siguiente modo:

1. términos que aparecen en los diccionarios generales con la marca de americanismo o argentinismo;

2. términos que solamente aparecen en los diccionarios propios del español argentino;

3. extranjerismos.

Se le dedica un apartado a los extranjerismos por la diferente utilización de ellos en países americanos con respecto al español peninsular. Como hemos comentado anteriormente, por varias razones, entre ellas geográficas, algunos países, como Argentina, son dados a utilizar mayor cantidad de extranjerismos con respecto a España $^{11}$. De esta manera, estos términos suponen otro tipo de diferenciación entre zonas hispanohablantes. Aunque no sean términos propios del español, cada zona hispanohablante hace un uso específico de ellos; muchas veces con grafías e incluso significados distintos.

Finalmente, agruparemos los términos según al ámbito temático hotelero al que pertenezcan. No tenemos que perder de vista que este trabajo representa un estudio léxico encaminado al discurso hotelero; por ello, resulta significativo saber a qué ámbito léxico hotelero pertenece el término o, al menos, saber en qué apartado de la web hotelera se utiliza. Por ello, incluiremos un apartado final con este tipo de clasificación.

${ }^{11}$ Aunque hay que tener en cuenta que, en menor medida, en las webs hoteleras españolas también se utilizan más extranjerismos que en otros ámbitos por recurrir a un discurso uniforme y persuasivo. 


\section{ANÁLISIS DEL CORPUS Y RESULTADO ${ }^{12}$}

2. 1. Términos que aparecen en los diccionarios generales con marca de Am. o Arg.: RAE (2001) y RAE (2005)

1. Banana:f.Arg., Col., Ec., Par. y Ur. plátano (planta musácea).

2. Bañadera: f. Am. Baño (pila). 2.f. Arg. y Ur. Ómnibus descubierto en el que se realizaban paseos o excursiones.

3. Cabotaje: $m$. Arg. Transporte aeronáutico mediante pago, entre puntos de un mismo Estado.

4. Calefaccionar: tr. Arg., Chile y Ur. Templar un ambiente mediante aparatos que generan calor.

5. Cancha: f. Am. Terreno, espacio, local o sitio llano y desembarazado.

6. Celular: $m$. Am. Teléfono móvil.

7. Desayunador: m. Méx. Habitación con mesa y sillas, cercana a la cocina, para tomar comidas ligeras.

8. Discado: $m$. del verbo discar Arg., Hond. y Ur. Acción de discar (marcar los números de teléfono).

9. Edilicia: adj. Arg., Bol., Col., Par., R. Dom. y Ur. Perteneciente o relativo a las obras o actividades de carácter municipal, especialmente las relacionadas con la edificación.

10. Extinguidor: m. Am. Extintor (aparato para extinguir incendios).

11. Faldeo: m. NO Arg. y Chile. Falda (parte baja de un monte).

12. Mate: $m$. Arg., Bol., Chile y Ur. Recipiente donde se toma la infusión de yerba mate, hecho de una calabaza pequeña o de otra materia.

13. Mucamo/a: $m$. y f. Arg. y Bol. En hospitales y hoteles, persona encargada de la limpieza.

14. Pava: f. Arg. y Par. Recipiente de metal o hierro esmaltado, con asa en la parte superior, tapa y pico, que se usa para calentar agua.

15. Payador: m. Arg., Bol., Chile y Ur. Cantor popular que, acompañándose con una guitarra y generalmente en contrapunto con otro, improvisa sobre temas variados.

16. Pileta: f. Arg., Bol. y Ur. Piscina (estanque para la natación). También abrevadero.

17. Placard: Armario empotrado donde se guarda la ropa y otros objeto.

18. Quincho: m. Arg., Par. y Ur. Cobertizo con techo de paja sostenido solo por columnas, que se usa como resguardo en comidas al aire libre.

19. Reposera: f. Arg., Par. y Ur. Tumbona (silla con largo respaldo).

${ }^{12}$ Los términos aparecen con la definición del diccionario del que han sido extraídos. Se ha seleccionado la acepción que correspondía al ejemplo extraído de la página web. Por otro lado, el orden de las entradas es alfabético.

NORMAS. REVISTA DE ESTUDIOS LINGÜÍSTICOS HISPÁNICOS, NÚMERO 3 (AÑO 2013):

http://www.uv.es/normas

(ISSN 2174-7245) 
20. Rotafolio: $m$. Hond. Atril en que se colocan hojas grandes de papel para escribir o dibujar durante una clase, charla o conferencia.

21. Rotisería: f. Á. R. Plata y Chile. Tienda donde se venden comidas para llevar, especialmente asados, quesos y fiambres.

22. Subte: $m$. Arg. Metropolitano (tren subterráneo).

23. Vacacionar: intr. Am. Cen., Arg., Chile, Méx., Ur. y Ven. Pasar las vacaciones.

24. Zapallo: adj. coloq. Arg. y Ur. Tonto (falto de entendimiento o razón). 2. m. coloq. Arg. y Ur. En el fútbol, gol.

Como podemos observar, todas estas voces son marcadas como americanismo o argentinismo. Aunque sean voces que aparecen en un diccionario del español general, debido a esta marca de americanismo, muchas de ellas no las utilizamos en el español peninsular, salvo alguna voz con extensión internacional, como sería el caso de mate; palabra extendida y conocida internacionalmente, aunque sea propia de la zona del Río de la Plata.

Hay otras palabras que aunque no las usemos, sí las reconocemos, nuevamente, por su extensión. Un caso claro es el de cancha, aunque en la mayor parte del español peninsular utilizamos campo, reconocemos cancha gracias, entre otras cosas, al fútbol argentino. De hecho, en la liga española de fútbol es frecuente tener jugadores y entrenadores argentinos y uruguayos que utilizan este término y que lo difunden continuamente en los medios de comunicación españoles. Otro caso es el de celular, término que hace referencia al teléfono móvil, referencia mayoritariamente peninsular, que aparece, una vez más, por su extensión geográfica.

Por otra parte, la mayoría de estos términos aparecen también en el Diccionario del habla de los argentinos y El gran diccionario de los argentinos. Pero el hecho de que aparezcan en diccionarios del español general, como por ejemplo en RAE (2001), significa que tienen una mayor extensión que si apareciesen solamente en los diccionarios específicos.

Muchas de estas palabras tienen equivalentes en el español peninsular bastante evidentes como:

- banana: plátano;

- bañadera: baño;

- calefaccionar: calentar;

- cancha: campo;

- celular: móvil;

- discar: marcar (los números del teléfono);

- extinguidor: extintor;

- faldeo: falda;

- pava: calentador;

- pileta: piscina; 
- reposera: tumbona;

- rotafolio: atril;

- subte: metro.

Sin embargo, hay otros términos en los que resulta algo más complejo encontrar una palabra que actúe como sinónimo. Sería el caso de edilicia, adjetivo que equivale a del edificio o de la construcción; desayunador: 'habitación para el desayuno'; cabotaje: 'transporte entre puertos'; rotisería sería una especie de restaurante donde se vende comida para llevar, en español peninsular se suele usar la denominación de comidas para llevar para el término que hace referencia al recinto que lleva a cabo la actividad de este servicio; mucamo/a sería hombrelmujer de la limpieza pero específico para hospitales y hoteles; vacacionar equivaldría a pasar las vacaciones, es cierto que en español peninsular contamos con veranear, pero este término es específico solamente para las vacaciones de verano; y quincho como 'cobertizo de paja entre dos columnas en comidas al aire libre'.

2. 2. Términos que aparecen en los diccionarios específicos de Argentina: el Diccionario del habla de los argentinos $y \mathrm{El}$ gran diccionario de los argentinos

1. Aerosilla: $f$. Sistema de transporte compuesto por uno o más asientos suspendidos de un cable de metal, que se utiliza para recorrer espacios aéreos en montañas o sierras: Montaron una nueva aerosilla en el centro de actividades de montaña.

2. Agendar: agendar tr. Anotar o registrar algo en una agenda: Ya mismo agendo la invitación. / Se agendó el compromiso para el martes.

3. Avistaje: $m$.Vista de algo o alguien a la distancia: un buen lugar para el avistaje de fauna.

4. Check-in: $1 \mathrm{~m}$. Proceso mediante el cual una persona se registra para obtener una habitación en un establecimiento hotelero: En ese hotel el check-in es a partir de las diez de la mañana. $\S 2 \mathrm{~m}$. Proceso mediante el cual una persona anuncia su presencia y confirma que se embarcará en algunos medios de transporte: Trabaja en el sector de check-in del aeropuerto. I Se accede al andén, previo check-in.

5. Check-out: (chekáut) $m$. Proceso de salida de un establecimiento hotelero, con la correspondiente liquidación de la cuenta de gastos: El horario de check-out está sujeto a la disponibilidad de habitaciones.

6. Churrasquera: Plancha de hierro que se usa para cocinar churrascos u otros alimentos: una churrasquera recubierta con teflón.

7. Espumante: espumante 1 adj. Que tiene o genera mucha espuma: Era un mar espumante. I Servir el plato acompañado de una cerveza muy espumante y helada. 
8. Kitchenette: (también kitchinette) (kíchenet o kichenét) $1 f$. Cocina eléctrica o a gas sin horno que consta de tres o cuatro pequeñas hornallas, se utiliza generalmente en espacios reducidos: Compraron una kitchenette para la cocina. $\S 2 f$. Espacio pequeño de una oficina o un departamento que está destinado a la cocina y está integrado al mismo ambiente: Las cabañas cuentan con un dormitorio matrimonial, baño completo y kitchenette.

9. Lenga: $1 \mathrm{f}$. Árbol fagáceo (Nothofagus pumilio) que mide treinta metros de altura. Sus hojas en otoño cambian de verde a rojo y luego a amarillo. Es resistente al frío y crece en los Andes patagónico-fueguinos. § $2 f$. Madera de la lenga [1] que se utiliza en carpintería y mueblería: sillas de lenga.

10. Loft: $m$. Departamento que consta de un único ambiente muy grande y sin divisiones, generalmente con mucha luz natural: Compró un loft en el segundo piso de lo que era una antigua fábrica. NOTA: el plural es loft o lofts: decoración de lofts / loft luminosos.

11. Microcentro: $m$. Área delimitada del centro de una ciudad, donde se lleva a cabo una gran cantidad de actividades comerciales, administrativas y de gobierno: Circular por el microcentro en auto es cada vez más difícil.

12. Notebook: (nout buk) $f$. Computadora personal de pequeño tamaño que integra en un mismo volumen la pantalla y el teclado: Me compré una notebook en cuotas.

13. Outlet: (áutlet o áulet) $1 \mathrm{~m}$. Establecimiento en el que se venden productos directamente de la fábrica y a precios rebajados, muchas veces por ser de temporadas anteriores o por tener alguna falla: los outlets de la avenida Córdoba. $\$ 2 \mathrm{~m}$. Venta de uno o varios productos a precios rebajados, generalmente temporaria y a causa de una renovación del stock: En ese local hay un outlet de zapatos. I Quiero aprovechar que la ropa de invierno está en outlet.

14. Pinotea: $f$. Madera empleada en carpintería que se obtiene de algunas especies de pino que se caracterizan por tener mucha resina: un piso de pinotea.

15. Pub: (pab) m. Bar donde se venden y se consumen bebidas, especialmente alcohólicas, y en ocasiones algunas comidas rápidas, en el que suele haber música ambiental: La vez pasada nos encontramos en un pub del centro.

16. Satelital: Perteneciente o relativo a los satélites artificiales, en especial a los de telecomunicaciones.

17. Servicio remís o remise: Automóvil con chofer, cuyo servicio, efectuado por horas o kilómetros de recorrido, se contrata en una agencia. / remisero (chofer).

18. Tercerizar: tr. Referido a una empresa o un organismo, poner en manos de otra empresa, un particular o una entidad prestadora de servicios la responsabilidad de ocuparse de una tarea determinada: Durante su mandato, YPF redujo personal, tercerizó procesos y pasó a tener solamente cinco mil empleados en todo el país. 
19. Toallón: $m$. Toalla grande que se usa para secarse el cuerpo: un toallón con capucha para la playa.

20. Toilette: (tualét) $1 \mathrm{~m}$. Habitación de una casa o un departamento en donde hay un inodoro y un lavatorio: Las visitas usan el toilette.

21. Tránsfer: $m$. Transporte de pasajeros, especialmente de turistas: El hotel ofrece un tránsfer.

22. Trekking: $m$. Deporte que consiste en recorrer a pie largas distancias, en terrenos generalmente irregulares: unas zapatillas de trekking / un circuito de trekking.

23. Wok: (guok o uok) $m$. Sartén de origen chino más profunda que las comunes: un plato de verduras al wok.

En el listado anterior observamos muchos términos que resultarían desconocidos para un hablante peninsular, como por ejemplo: churrasquera, remís/remise, agendar (con el significado de anotar algo en la agenda) o tercerizar. Este hecho refleja la especificidad de estos términos como propios de una zona específica, en este caso, el español de Argentina.

Por otro lado, hay que tener en cuenta que muchos de estos términos son extranjerismos. Es cierto que los extranjerismos van a tener un grupo independiente en este trabajo. Sin embargo, se ha decidido incluir algunos como toilette, check in, check out ${ }^{13}$ o notebook en este grupo por ser términos que no aparecen en los diccionarios generales del español y sí en los específicos seleccionados del español de Argentina.

\subsection{Extranjerismos}

1. Aguagym: gimnasia acuática.

2. Amenities: servicios, comodidades.

3. Baguette: baguete, barra de pan alargada.

4. Buffet: 1. m. bufé (comida). 2. m. Arg. bufé (mesa). 3. m. Arg. (local).

5. Caipirinha: caipiriña, tipo de cóctel.

6. Cama king: cama del rey, grande.

7. Cama queen: cama de la reina.

8. Cama twin: cama doble.

9. Camping: campin/campamento.

10. Capuccinno: café con espuma.

11. Catering: servicio de suministro de comidas y bebida.

12. Champagne: champán.

13. Chef: jefe de cocina.

14. Check in: registro en el hotel a la llegada.

15. Check out: salida del hotel. académicos.

13 Aunque sí puedan aparecer en corpus del español peninsular, pero no en los diccionarios 
16. Citytour: vuelta a la ciudad.

17. Cocktail: cóctel.

18. Coffee break: descanso para café.

19. Croissant: cruasán.

20. Deck: cubierta, cubrecama.

21. Family suites: habitación familiar.

22. Free: gratis.

23. Gym: gimnasio.

24. Junior (suite): júnior ${ }^{14}$.

25. Jet-lag: trastorno o malestar producido por un viaje en avión con cambios horarios considerables.

26. Kayak: cayac, embarcación deportiva.

27. Living: cuarto de estar.

28. Lobby: vestíbulo de un hotel.

29. Mozzarella: queso de búfala italiano.

30. Package: bulto, equipaje.

31. Pet friendly: establecimiento que permite mascotas.

32. Rafting: balsismo.

33. Rally: rali.

34. Rappel: rápel/rapel, descenso rápido en montañismo.

35. Restaurant: restaurante.

36. Room: habitación.

37. Sándwich: emparedado.

38. Service: servicio.

39. Shopping: centro comercial, zona de compras.

40. Show musical: espectáculo musical.

41. Sit-stress: estrés del sentado.

42. Snack: aperitivo.

43. Snowboard: deporte de nieve (tabla de nieve).

44. Sommier ${ }^{15}$ : estructura, en general con resortes en su interior, que tiene un contorno rígido y patas y está forrada de tela gruesa, sobre la que se apoya un colchón.

45. Spa: balneario.

46. Staff: personal de la empresa.

47. Suite: en los hoteles, conjunto de sala, alcoba y cuarto de baño.

48. Tea time: tiempo de té, el espacio del té.

49. Whisky, whiskey: güisqui.

50. Wi-fi: conexión inalámbrica de internet.

51. Yacuzzi: bañera para hidromasaje.

52. Yogurt: yogur.

\footnotetext{
${ }^{14}$ RAE (2005): júnior; RAE (2010): yúnior.

${ }^{15}$ En RAE (2001): somier.
} 
Como hemos comentado ya, es evidente el mayor uso de extranjerismos en el español argentino con respecto del español peninsular. Los motivos geográficos y estilísticos, también ya mencionados, son obvios en su aparición. Las páginas web hoteleras, muchas veces, aunque cuentan con términos patrimoniales equivalentes, optan por estos términos reconocibles más fácilmente internacionalmente. Esto también sucede en el español peninsular como señala Sanmartín (2011a: 349):

En suma, el anglicismo triunfa en el discurso turístico español de promoción de hoteles a ambos lados del Atlántico. Eso sí, los anglicismos concretos pueden coincidir o pueden en cada zona relexificar un ámbito distinto.

Hay términos que un cliente espera encontrar, y entiende perfectamente cuando entra en una web hotelera, como por ejemplo: yacuzzi, suite, cama king, cama queen, cama twin, lobby, amenities, check in, check out, family suites, restaurant, staff, room, service, catering. Por su especificidad hotelera, la mayoría de estos términos no aparece en los diccionarios de español general seleccionados. Sí existen algunas excepciones que son recogidas por RAE (2005) como sería el caso de lobby, staff, yacuzzi y catering:

- Lobby: cuando significa 'vestíbulo de un hotel y de otros establecimientos como cines, teatros, restaurantes, etc., especialmente si es grande', puede sustituirse por la voz española vestíbulo.

- Staff: voz inglesa que se usa ocasionalmente en español con los sentidos de 'conjunto de los trabajadores fijos de un organismo o empresa' y, en especial, 'conjunto de las personas que ocupan los puestos de mayor relieve en una organización o empresa'. Es anglicismo evitable, pues, para el primer sentido, el español cuenta con términos como personal o plantilla.

- Yacuzzi: adaptación gráfica propuesta para la voz inglesa jacuzzi, 'bañera dotada de un sistema para hidromasaje'. Se trata en origen de una marca registrada, que procede del apellido de quienes inventaron y comercializaron este sistema. El anglicismo puede también sustituirse por el equivalente español (bañera de) hidromasaje.

- $\quad$ Catering: catering $\rightarrow$ cáterin. Adaptación gráfica propuesta para la voz inglesa catering, 'servicio de suministro de comidas y bebidas a aviones, trenes, colegios, etc.'.

Si estos términos aparecen en RAE (2005) es por su interés gráfico. El Diccionario distingue entre extranjerismos necesarios (porque no tienen un equivalente en español) y extranjerismos innecesarios (porque sí tienen un equivalente en español). Para el grupo de los necesarios, lo que se propone es una adaptación gráfica al español en todas sus facetas, sería el caso de catering, anglicismo que no tienen un término que lo sustituya 
en español, por ello, se opta por la adaptación gráfica cáterin. También yacuzzi, que opta por la adaptación yacusi.

Por otro lado, tenemos los extranjerismos innecesarios como lobby o staff, que se consideran precisamente innecesarios por contar con los equivalentes vestíbulo y personal.

Otros casos son los de show, rafting y chef como extranjerismos innecesarios, por lo que sus equivalentes son: espectáculo, balsismo, jefe de cocina, y los extranjerismos necesarios yogurt, rally, champagne, rappel, camping, junior, whisky, croissant, sandwich, baguette, mozzarella, cocktail y buffet con sus respectivas adaptaciones: yogur, rali, champán, rápel, campin, yunior, güisqui, cruasán, sándwich, baguete, mozarela, cóctel y bufé o bufete.

\section{CLASIFICACIÓN POR ÁMBITO TEMÁTICO HOTELERO}

- Habitaciones: placard, reposera, toilette, toallón, deck, sommier, amenities, junior suite, family suites, room.

- Instalaciones: desayunador, cancha, bañadera, extinguidor, pinotea, living, kitchenette, gym, lobby, spa.

- Restauración: banana, pava, mate, quincho, wok, espumante, churrasquera, buffet, cocktail, snack, capuccinno, whisky, croissant, sándwich, caipirinha, baguette, mozzarella, restaurante, champagne, tea time, catering, yogurt, coffee break.

- Servicios: pileta, mucama, calefaccionar, tránsfer, check in, check out, yacuzzi, wifi, free, staff, service.

- Zonas de ocio: rotisería, pub, outlet, microcentro, shopping, show musical.

- Actividades deportivas: trekking, snowboard, rafting, aguagym, rappel, rally, kayak.

- Transporte y viajes: cabotaje, faldeo, subte, vacacionar, remís, remisero, aerosilla, avistaje, jet-lag, sit-stress, package, camping, citytour.

- Otros: discado, celular, rotafolio, payador, zapallo, satelital, notebook, lenga, agendar, loft, tercerizar.

\section{CONCLUSIÓN}

Con este trabajo hemos observado las posibilidades y la inmediatez que ofrece internet, en concreto las páginas web hoteleras, a la hora de realizar un trabajo terminológico de investigación. Ante esta facilidad, el número de textos ha aumentado considerablemente con respecto al uso publicitario en papel que se venía usando en este ámbito. Además, el género turístico en sí se ve modificado, como señala Calvi (2010), 
por la integración de léxico de otros sectores, como por ejemplo la restauración, la cultura o la historia.

Por otro lado, no podemos olvidar el objetivo comercial que hay detrás de las páginas web turísticas y que influirá a la hora de seleccionar los términos. De hecho, muchas de las características del léxico turístico en este campo se ven afectadas por este fin, ya que se buscará aquel término que mejor armonice con este objetivo y, por lo tanto, que resulte más atractivo. Así pues, un anglicismo reconocido internacionalmente y que, además, esté de moda, puede ser mucho más atractivo que un término patrimonial que solo se entiende en determinadas zonas. De ahí que muchas veces se opte por un extranjerismo crudo en lugar de un término propio; sería el caso de show, rafting, room y chef en lugar de espectáculo, balsismo, habitación, jefe de cocina.

De este modo, la primera característica que encontramos en el léxico turístico argentino es la preferencia por determinados extranjerismos crudos en favor del objetivo comercial. De hecho, muchos de estos extranjerismos se sistematizan hasta el punto de poderlos encontrar tanto en el español peninsular como en el americano; por ejemplo, en la denominación de la habitación del hotel: king, queen, twin, suite, etc. Aunque es cierto que en el español argentino se suele tender todavía más al extranjerismo que en el español peninsular, especialmente a los anglicismos. También puede suceder que encontremos el uso de la forma patrimonial y el extranjerismo crudo dentro del mismo texto, como por ejemplo con room o habitación. Sin embargo, hay otros casos en los que el equivalente apenas se contempla, como en check in, check out o chef.

Además de esta preferencia por formas extranjeras, se puede rescatar el uso de términos patrimoniales argentinos (o del español de América); sería el caso de mate, mucama, bañadera, pileta, remís, quincho. Estos términos patrimoniales conviven con los extranjerismos citados anteriormente como señala Calvi (2006b). De esta forma se puede singularizar lo propio de un país, en este caso Argentina.

En el último punto, hemos podido observar cómo los diccionarios del español general como RAE (2001 y 2005) ya contemplan la variante americana e incluyen sus términos, es el caso de voces extraídas de las páginas web analizadas como pileta, bañadera, discar, subte, mate, celular, etc. Esto se debe al intento de las diferentes academias de la lengua española de mantener una uniformidad en la lengua española en todas sus variantes para evitar una posible fragmentación del idioma.

Aun así, no se puede evitar que haya términos que no aparezcan recogidos por estos diccionarios y, por lo tanto, tengamos que acudir a diccionarios más específicos como el Diccionario del habla de los argentinos o El gran diccionario de los argentinos para términos como kitchenette, tercerizar, agendar, toallón, microcentro, churrasquera o remís. En este caso, estos términos se reconocen como propios del español argentino. Así pues, podemos diferencia entre lo que oficialmente, y según la RAE (2001 y 2005), se considera español general o variante americana de uso extendido (mate, celular, etc.) y lo que se considera como español específico de una variante (caso de remís o churrasquera).

NORMAS. REVISTA DE ESTUDIOS LINGÜÍSTICOS HISPÁNICOS, NÚMERO 3 (AÑO 2013):

http://www.uv.es/normas

(ISSN 2174-7245) 
Otro dato que resulta llamativo es el hecho de encontrar la mayoría de estos términos específicos en los hoteles de categoría más baja. Así pues, cuanta mayor categoría tenga el hotel, menos tenderá a usar términos propios o específicos de la zona en cuestión. Por ello, gran parte del léxico propio lo hemos encontrado en los hoteles de una o dos estrellas.

En general, a pesar de esta variedad léxica que vendría provocada por la diferente categoría del hotel y que así podría fomentar la diversidad léxica, podemos observar la presencia de un léxico más o menos uniformado, como señala Sanmartín (2011a). La autora considera que hay más unidad que diversidad. Esto se debe a las pautas que se le exige a un producto, como podría ser el tipo de habitación o servicios de un hotel. Estos servicios tienen que ser reconocidos por el cliente cuando se acerca a una página web hotelera, ya que de lo contrario se podría poner en peligro la compra de un producto provocado por una confusión de términos.

Para finalizar, la revolución que supone internet en los ámbitos publicitarios no ha hecho más que empezar. La revolución tecnológica va a traer, y está trayendo, avances y nuevos campos que van a reforzar el fenómeno del márquetin no solo en el ámbito hotelero. Además de las webs, contamos también con las redes sociales y el desarrollo de las aplicaciones, tanto para ordenadores como para móviles o celulares. Estaremos atentos para recoger este factor, junto con una ampliación del estudio comparativo del español. En concreto, ya se está realizando un estudio contrastivo del léxico turístico que engloba diversos países del español de América y ciudades de la Península dentro del marco que supone internet (proyecto ya citado).

\section{REFERENCIAS BIBLIOGRÁFICAS}

ACADEMIA ARgENTINA DE LETRAS (2005): Diccionario del habla de los argentinos, Buenos Aires, Espasa.

AlCARAZ, Enrique et al. (2006): Diccionario de términos de turismo y de ocio. Inglés-Español, Spanish-English, Barcelona, Ariel.

ALEZA, Milagros (2000): «Nuevos enfoques metodológicos en la práctica lexicográfica hispanoamericana diferencial actual (sobre algunos diccionarios de los 90)», en Aleza, Milagros y Ángel López, eds., Estudios de Filología, Historia y cultura hispánica, Valencia, Universitat de València, 27-48.

ALEZA, Milagros (2005): «Sobre la delimitación de los americanismos léxicos a la luz de la lexicografía hispanoamericana actual», en Hernández Alonso, César, ed., Filología y lingüística. Estudios ofrecidos a Antonio Quilis, I, Madrid, CSIC, 655-674.

AlEZA, Milagros y José María EnguiTA (2002): El español de América, aproximación sincrónica, Valencia, Tirant Lo Blanch.

ARAGÓN, Marina et al. (2009): Diccionario de términos del turismo, FrancésEspañol, Espagnol-Français, Barcelona, Ariel. 
ARCARONS, Ramón et al. (2008): «La clasificación hotelera en la Unión Europea, un mercado poco común» [en línea]: <http,//www.estig.ipbeja.pt/ ac_direito/ClassHotUEMercadoPoco Comun.rtf >.

BLASCO, Albert, ed., (2006): Manual de gestión de producción de alojamientos y restauración, Madrid, Síntesis.

CABRÉ, Teresa (1993): La terminología. Teoría, metodología y aplicaciones. Barcelona, Antártida /Empúries.

CALVI, Maria Vittoria (2006a): Lengua y comunicación en el español del turismo, Madrid, Arco/Libros.

CALVI, Maria Vittoria (2006b): «El uso de términos culturales en el lenguaje del turismo, los hoteles y su descripción», en Calvi, Maria Vittoria y Luisa Chierichetti, eds., Nuevas tendencias en el discurso de especialidad, Berna, Peter Lang, 271-292.

CALVI, Maria Vittoria (2010): «Los géneros discursivos en la lengua del turismo: una propuesta de clasificación», Ibérica, 19 (Spring 2010), 9-32.

CALVI, Maria Vittoria y Giovanna MAPELli (2011): La lengua del turismo. Géneros discursivos y terminología, Berna, Peter Lang.

Clarín [en línea], El Gran diccionario de los argentinos, Argentina [en línea]: $<$ http,//www.clarin.com/diccionario>. [Consulta, 01/07/2013].

CRONIN, Michael (2000): Across the Lines. Travel, Language, Translation, Cork, Cork University Press.

ESTORNELL, María (2009): Neologismos en la prensa, criterios para reconocer y caracterizar las unidades neológicas, Valencia, Quadernos de Filologia, anejo 70, Universitat de València.

FREIXÀ, Judit et al. (2002): «La variación terminológica en las aplicaciones lexicográficas», en Actas del VIII Simposio Iberoamericano de Terminología, Unión Latina, Cartagena de Indias [en línea]: 〈www.upf.edu/pdi/dtf/teresa.cabre/docums/ca02ko.pdf >.

GALlego, Felipe y Ramón PEYROLÓN (2009): Diccionario de hostelería. Hotelería y turismo, restaurante y gastronomía, cafetería y bar, Madrid, Paraninfo.

MORENO, Francisco (1998): Principios de sociolingüística y sociología del lenguaje, Barcelona, Ariel.

REAL ACADEMIA ESPAÑOla (2001): Diccionario de la lengua española, Madrid, Espasa.

REAL ACADEMIA ESPAÑOLA Y ASOCIACIÓN DE ACADEMIAS DE LA LENGUA ESPAÑOLA (2005): Diccionario panhispánico de dudas, Madrid, Santillana.

REAL ACADEMIA ESPAÑOla Y ASOCIACIÓN DE ACADEMIAS DE LA LENGUA ESPAÑOLA (2010): Diccionario de americanismos, Madrid, Santillana.

REAL ACADEMIA ESPAÑOLA Y ASOCIACIÓN DE ACADEMIAS DE LA LENGUA ESPAÑOLA (2010): Ortografía de la lengua española, Madrid, Santillana.

SANMARTíN SÁEZ, Julia (2011a): «Unidad y variación en el español del turismo, las páginas web de promoción de hoteles de España y Chile», en Calvi, Maria Vittoria y Maria Giovanna Mapelli, eds., La lengua del turismo, Géneros discursivos y metodología, Bern, Peter Lang, 335-357. 
SANMARTÍN, Julia y GONZÁleZ, Virginia (2011b) «Corpus, diccionarios y discurso turístico: el proyecto de diccionarios bidireccionales español-francés-inglésárabe», en Maruenda-Battaller, Sergio y Begoña Clavel-Arroitia, eds., Multiple Voices in Academic and Professional Discourse, Cambridge Scholars Publishing, 392-403.

SANMARTÍN SÁEZ, Julia (2012): Discurso turístico e internet, Madrid, Iberoamericana/Vervuert.

SECO, Manuel et al. (1999): Diccionario del español actual, Madrid, Aguilar. 